\title{
The effect of yield stress on the performance of viscosity regularization
}

\author{
A. Ahmadi \\ Dept. of Mechanical, Industrial \& Aerospace Engineering \\ Concordia University \\ Montreal, Canada \\ am_ahma@encs.concordia.ca
}

\author{
I. Karimfazli \\ Dept. of Mechanical, Industrial \& Aerospace Engineering \\ Concordia University \\ Montreal, Canada \\ ida.karimfazli@concordia.ca
}

\begin{abstract}
In this study we investigate the effect of yield stress on the performance of viscosity regularization models in predicting unsteady flow of yield stress fluids. Viscosity regularization is popular for its simplicity of implementation into CFD codes and relatively low computational cost; however, the discussion on the limitations of this method in predicting the flow behavior has remained qualitative. We present a quantitative comparison between regularization and exact rheology in predicting natural convection of the Bingham fluids in a square cavity with differentially heated sidewalls. We consider a range of Bingham numbers and compare viscosity regularization results with augmented Lagrangian solutions. We show that the accuracy of viscosity regularization declines as the Bingham number increases. We also show that for high yield stress values the computational cost of viscosity regularization increases significantly.

Index Terms-Yield stress fluids, Viscosity regularization, Biviscosity, Unsteady flow
\end{abstract}

\section{INTRODUCTION}

Yield-stress fluids, YSF, behave like solids under any applied shear stress below a threshold called the yield stress. If the applied stress exceeds the yield stress, YSF flow like a fluid. In nature, avalanches, mud flow or landslides, lava flows and many biological substances such as mucus can display similar behavior. In industrial settings, chocolate confections, the cement slurry used in buildings or paints, pulp suspensions in paper production, pharmaceutical and cosmetic products involve the YSF (1)(2). Bingham fluids, named after Bingham (3), are described as YSF with the following ideal relation between the shear stress and the strain rate:

$$
\text { if } \quad \hat{\tau}<\hat{\tau}_{y} \quad \hat{\dot{\gamma}}_{i j}=0 \text {, otherwise } \quad \hat{\tau}_{i j}=\left[\hat{\mu}+\frac{\hat{\tau}_{y}}{\hat{\dot{\gamma}}}\right] \hat{\dot{\gamma}}_{i j}
$$

where $\hat{\dot{\gamma}}_{i j}$ and $\hat{\tau}_{i j}$ are the rate of strain and the deviatoric stress tensors. $\hat{\dot{\gamma}}$ and $\hat{\tau}$ are the second invariants of the rate of strain and deviatoric stress tensors:

$$
\begin{aligned}
& \hat{\dot{\gamma}}=\sqrt{\frac{1}{2} \hat{\dot{\gamma}}_{i j} \hat{\dot{\gamma}}_{i j}} \\
& \hat{\dot{\tau}}=\sqrt{\frac{1}{2} \hat{\tau}_{i j} \hat{\tau}_{i j}}
\end{aligned}
$$

$\hat{\tau}_{y}$ and $\hat{\mu}$ are the yield stress and plastic viscosity. The effective viscosity, $\hat{\eta}_{e}$, therefore, becomes:

$$
\hat{\eta}_{e}=\hat{\mu}+\frac{\hat{\tau}_{y}}{\hat{\dot{\gamma}}} .
$$

Other popular constitutive laws for YSF are Herschel-Bulkley and Casson models that have different definitions of effective viscosity. Although these constitutive models do not describe the exact behavior of YSF, they are convenient in practice. The effective viscosity of Bingham, Herschel-Bulkley and Casson models are similarly unbounded and discontinuous at $\hat{\dot{\gamma}}=0$ and this brings about theoretical and numerical challenges: (i) the stress fields within plug regions are indeterminate, (ii) the effective viscosity is singular at the plug boundaries (where $\hat{\tau}=\hat{\tau}_{y}$ ) and (iii) the shape and size of the unyielded regions are also not known a priori. This is usually remedied through the use of (a) augmented Lagrangian methods $(A L)$ or (b) viscosity regularizations models $(V R)$. The augmented Lagrangian methods, introduced by Glowinski (4), use the variational formulation of the Navier-Stockes equations and an optimization algorithm to perform numerical simulations of flow of YSF. Numerical predictions based on AL can accurately identify unyielded regions; they cannot, however, be easily integrated in most commercially available CFD codes and computation times can be prohibitively long (5). VR, first used by Glowinski (6), facilitates numerical simulations by estimating the effective viscosity using a bounded function: a regularized model represents a strongly shear-thinning but purely viscous fluid. VR can be easily implemented in commercial CFD codes and are generally faster than AL (1). Commonly used variations of VR are the models proposed by Bercovier and Engelman (7), Papanastasiou (8), and Biviscosity model, developed by (9). Bi-viscosity model estimates the effective viscosity using a bilinear curve:

$$
\hat{\eta}_{e}= \begin{cases}m \hat{\mu} & \hat{\dot{\gamma}} \leq \hat{\dot{\gamma}}_{c r} \\ \hat{\mu} & \text { Otherwise }\end{cases}
$$

Here $\hat{\mu}_{y}=m \hat{\mu}$ is the yield viscosity and $\hat{\dot{\gamma}}_{c r}=\hat{\tau}_{y} /\left(\hat{\mu}_{y}-\hat{\mu}\right)$ is the critical strain rate. $m$ is the regularization parameter and is inherently dimensionless. The choice of this parameter determines the accuracy of the regularization model in estimating 


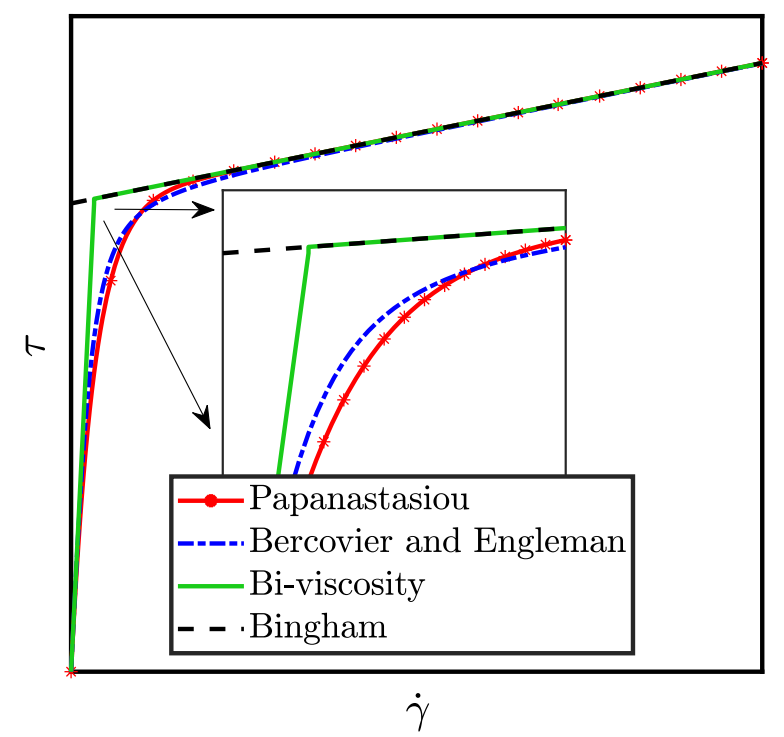

Fig. 1. Illustration of viscosity regularization models and Bingham model. The regularization parameter is chosen such that $\frac{\left[\hat{\eta}_{e}\right]_{\hat{\gamma}=0}}{\left[\hat{\eta}_{e}\right]_{\hat{\gamma}=\infty}}=100$.

the Bingham constitutive law. See Fig. 1 for an illustration of the different regularization models.

Generally, the difference between VR and Bingham model is maximum for small strain rates and it diminishes as $\dot{\gamma} \rightarrow \infty$. The convergence of the predictions based on VR and the exact Bingham rheology has been discussed by (10). They provided error bounds for the difference between the results of VR and the exact Bingham model. They have shown that when the shear stresses is close to the yield stress in relatively large part of the flow domain, VR errors are the largest. Turan et al utilized bi-viscosity model to study the steady state natural convection in a square cavity with differentially heated side walls. Karimfazli et al (11) studied the temporal evolution of the flow in the same problem, utilizing the AL.

The limitations of VR have been primarily considered qualitatively and in predicting steady flows. The goal of this study is to investigate the effect of VR on the accuracy of unsteady flows of YSF. We present a quantitative comparison of AL and VR in predicting the buoyancy-driven flow of YSF in a square cavity subject to differentially heated sidewalls. In $\S$ III-C, we validate our results in comparison with numerical solutions of (12) and (11). In III, we present unsteady flow development based on VR and discuss the accuracy and numerical complications. §IV summarizes our observations and draws the conclusions.

\section{Problem Setup}

We consider two-dimensional flow between two differentially heated vertical walls confined to two adiabatic horizontal walls $(\partial \hat{T} / \partial \hat{x}=0)$ in a square geometry. Fig. 2 shows the

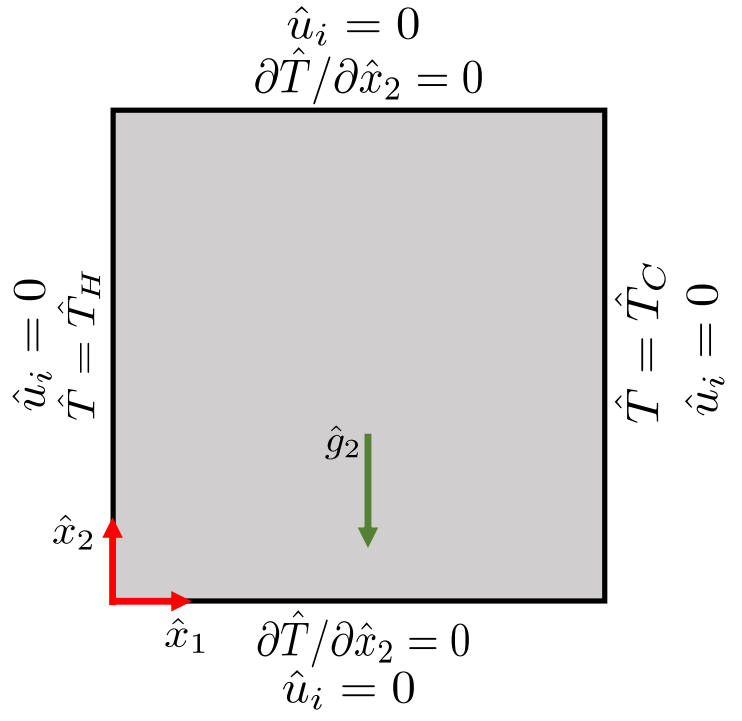

Fig. 2. Problem setup and boundary conditions. $\hat{T}$ is temperature and $\hat{u}_{i}$ is the velocity in $i$ th direction where $i, j=1,2$ and $\left(x_{1}, x_{2}\right)=(x, y)$.

schematics of boundary conditions. Left wall is maintained at a constant hot temperature, $T_{H}$ and right wall has a constant cold temperature, $T_{C},\left(T_{C} \leq T_{H}\right)$. No-slip boundary conditions is applied on all the boundaries of the cavity. Initially (at $t=0$ ), the Bingham fluid is at rest and has a linear temperature distribution. This is representative of the steady purely conductive state.

\section{A. Formulation}

In this paper we denote dimensional quantities with^symbol and dimensionless quantities without. Assuming Boussinesq's approximation, three dimensionless groups govern the flow dynamics:

$$
\operatorname{Pr}=\frac{\hat{\nu}}{\hat{\kappa}}, \quad R a=\frac{\hat{g} \hat{\beta} \Delta \hat{T} \hat{L}^{3}}{\hat{\nu} \hat{\kappa}}, \quad B=\frac{\hat{\tau}_{y}}{\hat{\rho}_{0} \hat{g} \hat{\beta} \Delta \hat{T} \hat{L}}
$$

Here $\hat{\mu}, \hat{\kappa}, \hat{\beta}, \hat{g}, \hat{L}$ and $\Delta \hat{T}$ are the fluid's momentum diffusivity, thermal diffusivity and coefficient of thermal expansion, acceleration due to gravity, the cavity width and the temperature difference between the two sidewalls $\left(\hat{T}_{H}-\hat{T}_{L}\right)$, respectively. $\hat{\rho}_{0}$ is the density evaluated at reference temperature, $\hat{T}_{0}=\left(\hat{T}_{H}+\hat{T}_{C}\right) / 2 . \operatorname{Pr}$ is the Prandtl number representing the ratio of momentum diffusivity to thermal diffusivity. Rayleigh number, $R a$, represents the balance of the destabilizing effect of buoyancy stresses to the stabilizing effect of viscosity. Bingham number, $B$, represents the ratio of the yield stress, $\hat{\tau}_{y}$ to buoyancy stresses. If the Bingham number exceeds a critical limit $B_{c r}$, the steady state becomes motionless. Karimfazli et al (11) demonstrated that $B_{c r}$ is independent of other dimensionless numbers and only depends on the geometry. They calculated $B_{c r}=0.03125$ for square cavity. 
Non-dimensional governing equations (momentum, continuity and energy equations) become:

$$
\begin{aligned}
& \frac{1}{\operatorname{Pr}} \frac{\partial u_{i}}{\partial t}+\frac{R a}{P r} u_{j} \frac{\partial u_{i}}{\partial x_{j}}=-\frac{\partial p}{\partial x_{i}}+\frac{\partial \tau_{i j}}{\partial x_{j}}+T \delta_{i 2} \\
& \frac{\partial u_{i}}{\partial x_{i}}=0 \\
& \frac{\partial T}{\partial t}+\operatorname{Rau}_{i} \frac{\partial T}{\partial u_{j}}=\frac{\partial^{2} T}{\partial x_{i} \partial x_{i}}
\end{aligned}
$$

We use a viscosity regularization of Bingham model known as bi-viscosity to relate the deviatoric stress tensor to the strain rate,

$$
\tau_{i j}= \begin{cases}m \dot{\gamma}_{i j}, & \text { if } \dot{\gamma} \leq \dot{\gamma}_{c r} \\ \left(1+\frac{B}{\dot{\gamma}}\right) \dot{\gamma}_{i j}, & \text { otherwise }\end{cases}
$$

Here the ratio of the yield viscosity $\left(\hat{\mu}_{y}\right)$ to the plastic viscosity $(\hat{\mu})$ is the regularization parameter $m=\frac{\hat{\mu}_{y}}{\hat{\mu}}$. The viscosity of the fluid switches from yield viscosity to plastic viscosity if the strain rate exceeds $\dot{\gamma}_{c r}=\frac{B}{m-1}$. We used $m=10^{3}$ for all the simulations presented in this paper. The dimensionless initial and boundary conditions are

$$
\begin{aligned}
& \left\{\begin{array}{l}
u_{i}(t=0, x, y)=0, \quad i=1,2 \\
T(t=0, x, y)=-x+1 / 2
\end{array}\right. \\
& \left\{\begin{array}{l}
u_{i}(t, x=0, y)=u_{i}(t, x=1, y)=0 \\
u_{i}(t, x, y=0)=u_{i}(t, x, y=1)=0, \quad i=1,2 \\
T(t, x=0, y)=1 / 2 \\
T(t, x=1, y)=-1 / 2
\end{array}\right.
\end{aligned}
$$

The dimensionless variables in the above equations are defined as

$$
\begin{aligned}
& x_{i}=\frac{\hat{x}_{i}}{\hat{L}}, \quad T=\frac{\hat{T}-\hat{T}_{0}}{\hat{T}_{H}-\hat{T}_{C}}, \quad u_{i}=\frac{\hat{\nu} \hat{u}_{i}}{\hat{g} \hat{\beta} \Delta \hat{T} \hat{L}^{2}}, \\
& t=\frac{\hat{\kappa} \hat{t}}{\hat{L}^{2}}, \quad \tau_{i j}=\frac{\hat{\tau}_{i j}}{\hat{\rho}_{0} \hat{g} \hat{\beta} \Delta \hat{T} \hat{L}}, \quad \dot{\gamma}_{i j}=\frac{\hat{\nu} \hat{\dot{\gamma}}_{i j}}{\hat{g} \hat{\beta} \Delta \hat{T} \hat{L}} .
\end{aligned}
$$

The purely conductive temperature profile, corresponding to the case where fluid is motionless everywhere in the domain, is simply $T=-x+0.5$. We also introduce $\theta=T+x-0.5$ as a measure of the advective heat transfer. $L^{2}$ norms of velocity, $\mathbf{u}$, and temperature, $\theta$ defined as

$$
\|\theta\|=\sqrt{\int_{\Omega} \theta^{2} d \Omega} \text { and }\|\mathbf{u}\|=\sqrt{\int_{\Omega} u^{2} d \Omega} .
$$

are used throughout this paper to illustrate development of the flow from the initial motionless state $(\|\theta\|=\|u\|=0)$.

\section{B. Numerical Method}

We solve equations (4 - 6) in a domain with $80 \times 80$ discrete control volumes, using the finite-volume based software FLUENT v19.2. We used second-order and fully implicit temporal formulation and iterate within each time step for the rest of the terms. The convergence criteria for the residuals of the above equations is set to $10^{-6}$. As for spatial discretization we use a second-order pressure interpolation and a second-order upwind scheme for convection terms. Pressure and velocity are coupled by segregated algorithm, SIMPLE and before each SIMPLE iteration the viscosity is updated following equation 3 , implemented into a user-defined function.

\section{Validation}

In table I, we compare our numerical results with (12) and (11). In table II, we present the mesh convergence of our numerical simulation. Note that $B n$ is the dimensionless number used by (12) for this problem.

$$
B n=\frac{\tau_{y}}{\mu} \sqrt{\frac{L}{g \beta \Delta T}}=B \sqrt{\frac{R a}{P r}}
$$

Table II shows that in comparison with the finest mesh $M 4$, the accuracy of the results for $V_{\max }$ were improved from $5.7 \%$ to $1.3 \%$ by changing grid size from $M 2$ to $M 3$. The average Nusselt number accuracy were also improved from $1.6 \%$ to $0.2 \%$. Therefore, $M 3$ is a reasonably fine mesh for this problem providing satisfactory accuracy and reasonable computational cost. Moreover, the augmented Lagrangian results presented in this paper were conducted on a $100 \times 100$ grid. Thus, we chose a similar mesh size to facilitate the comparison between AL and VR methods. Hence, unless otherwise specified, $M 3$ grid was used for the simulations presented in this manuscript.

\section{RESUlTS AND DISCUSSION}

We provide numerical simulations of the natural convection of Bingham fluid in a square cavity with differentially heated sidewalls. We mainly asses a performance of the regularized Bingham model (bi-viscosity) in retrieving the results achieved using the Bingham model. For this purpose, we compared our results with the work of (11). Their computations are based on augmented Lagrangian method. We investigated the flow development at $R a=10^{4}$ and $\operatorname{Pr}=1$ for fluids with different Bingham numbers to study the effect of yield stress on the accuracy of our simulations.

Fig. 3 depicts the evolution of the velocity norm as flow evolved from the initially motionless and purely conductive state to steady state (at $R a=10^{4}$ and $P r=1$ ). The results of bi-viscosity regularization are shown by a solid red curve (BV) and the augmented Lagrangian results by solid black curve

\begin{tabular}{ccccc}
\hline & & Present Study & $(11)$ & $(12)$ \\
\hline Bingham fluid & & & & \\
& $\overline{N u}$ & 1.5256 & 1.5269 & 1.5248 \\
$\operatorname{Pr}=7, B n=0.5$ & $V_{\max }$ & 8.9580 & 8.9441 & 8.9490 \\
& & & & \\
\hline Newtonian fluid & $\overline{N u}$ & 2.243 & 2.248 & 2.245 \\
& & & & \\
$\operatorname{Pr}=0.71, B n=0$ & $V_{\max }$ & 19.620 & 19.617 & 19.655 \\
& & & & \\
\hline
\end{tabular}

TABLE I: Comparison of simulation results with (11) and (12) at $R a=10^{4}$ 


\begin{tabular}{ccccc}
\hline & & Present Study & $(11)$ & $(12)$ \\
\hline \multirow{2}{*}{$M 2=40 \times 40$} & $\overline{N u}$ & 1.5017 & 1.5382 & 1.5109 \\
& $V_{\max }$ & 8.4453 & 8.8925 & 8.2614 \\
$M 3=80 \times 80$ & $\overline{N u}$ & 1.5234 & 1.5269 & 1.5224 \\
& $V_{\max }$ & 8.8393 & 8.9446 & 8.7900 \\
$M 4=160 \times 160$ & $\overline{N u}$ & 1.5256 & 1.5269 & 1.5248 \\
& $V_{\max }$ & 8.9580 & 8.9441 & 8.9490 \\
\hline
\end{tabular}

TABLE II: Comparison with Karimfazli et al. and Turan et al. for simulation of Bingham fluid ( $B n=0.5, B=$ 0.0132, $R a=10^{4}, \operatorname{Pr}=7$ ).

(AL). We observe similar qualitative evolution trends in both solutions for low to moderate Bingham numbers $(B \leq 0.015)$. In high Bingham number $(B=0.025)$ however, a gap is evident between unsteady (and steady) AL and BV results. To quantify the comparison, we defined the discrepancy of a variable of interest, $\phi$, between the AL numerical solution, $\phi_{A L}$, and BV numerical solution, $\phi_{B V}$, by $\delta(\phi)=\mid \phi_{B V}-$ $\phi_{A L} \mid / \phi_{A L}$. Fig. 4 illustrates the evolution of $\delta(\|u\|)$ and $\delta(\|\theta\|)$. Fig. 4 (a-d) show that when $B \leq 0.015$, the magnitude of $\delta(\|u\|)$ is mostly lower than $1 \%$ and it grows with the yield stress. This increase in $\delta(\|u\|)$ is more noticable at higher Bingham numbers. This substantial increase in $\delta(\|u\|)$, persists throughout the flow development. In fig. $4(\mathrm{e}-\mathrm{h})$ we observe similar loss of accuracy in predicting convective heat transfer $\theta$. This can be associated with the growth of the areas with sub-critical strain rates $\left(0 \leq \dot{\gamma} \leq \dot{\gamma}_{c r}\right)$ as $B$ approaches $B_{c r}$. Fig. 5 illustrates the areas of the sub-critical strain rates in the domain. Under the same buoyancy forces, the fluid with higher yield stress generally would produce lower strain-rates, thus, it is evident as the $B \longrightarrow B_{c r}$, the areas of the domain possessing sub-critical strain rate expand. Biviscosity model underestimates the shear stress of the Bingham model where $0 \leq \dot{\gamma} \leq \dot{\gamma}_{c r}$; this is the range where the regularized model is least accurate in estimating the exact rheology of a Bingham fluid. In fig. 5, we observe that the area with sub-critical strain rates expands substantially from $B \leq 0.015$ to $B=0.025$, this justifies the substantial increase in $\delta(\|u\|)$ and $\delta(\|\theta\|)$.

We also observed that increasing the Bingham number, significantly increases the computational cost of the problem. To illustrate this, Fig. 6 shows the decay of error in the governing equations 5 vs. iteration at an arbitrary time-step. Comparing the number of iterations needed for the Newtonian fluid $(B=0)$ and the Bingham fluids $(B>0)$ shows that the solver demands considerably more time and computational power to meet the convergence criteria upon the introduction of the yield stress. Evidently, increasing the Bingham number leads to a significant increase in the required iterations. All simulations follow a similar pattern for the parameter ranges considered in this study. In other words, the Bingham fluid with a higher yield stress requires more iterations to converge to the same residual.

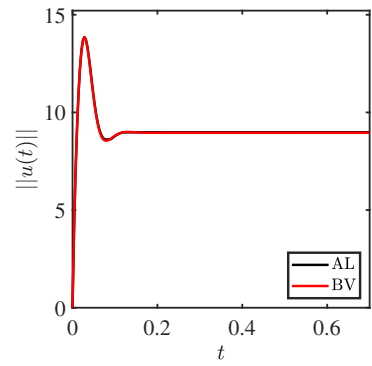

(a) $B=0.005$

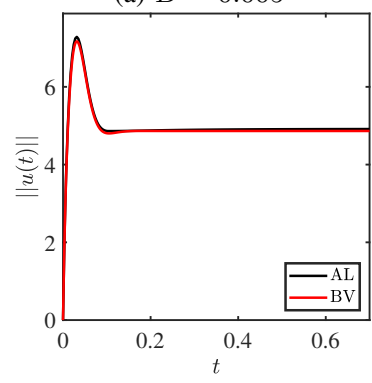

(c) $B=0.015$

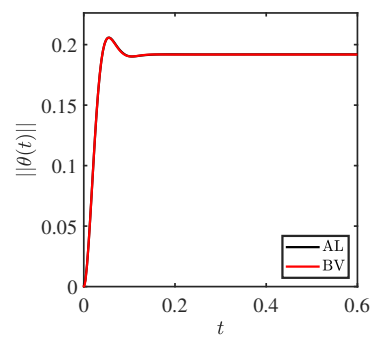

(e) $B=0.005$

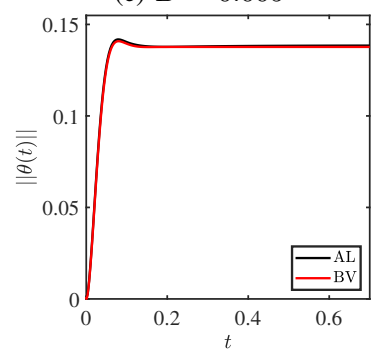

(g) $B=0.015$

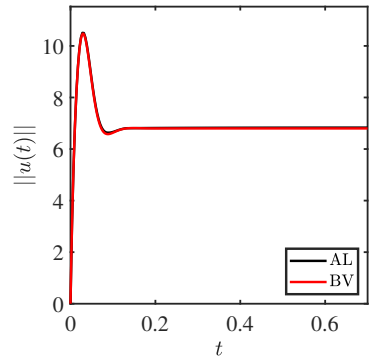

(b) $B=0.01$

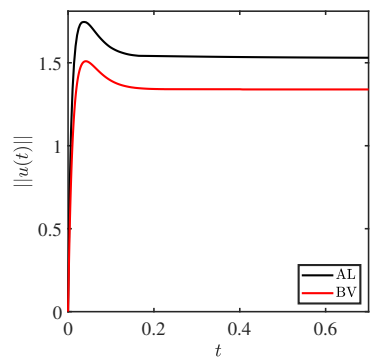

(d) $B=0.025$
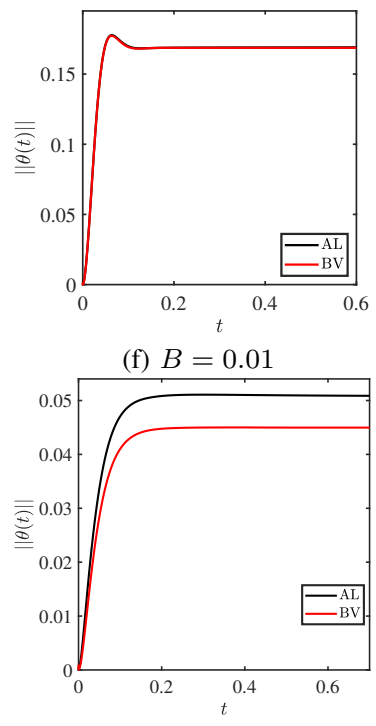

(h) $B=0.025$ (f) $B=0.01$

Fig. 3. The evolution of $(\mathrm{a}-\mathrm{d})$ velocity $\|u\|$ and $(\mathrm{e}-\mathrm{h})$ convective heat transfer $\|\theta\|$, for different Bingham numbers at $R a=10^{4}$ and $\operatorname{Pr}=1$.

\section{SUMMARY}

We have investigated the application of viscosity regularization in predicting unsteady flow of yield-stress fluids. The studied case was the natural convection inside a square cavity with differentially heated side walls. Having large but finite viscosity, regularized Bingham fluids flow under any stress, Bingham fluid in contrary doesn't flow unless the yield stress is exceeded. This feature is more pronounced when the flow produces stresses near the yield stress (strain-rate is near zero). The aim of this study has been to explore how this approximating the rheology by regularization can affect the predicted unsteady flow and to 


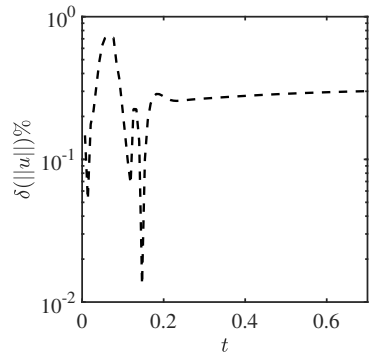

(a) $B=0.005$

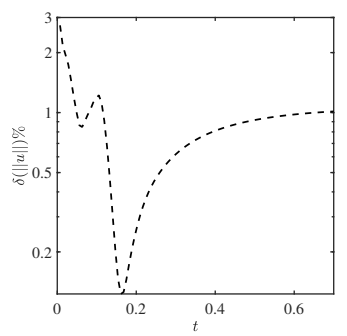

(c) $B=0.015$

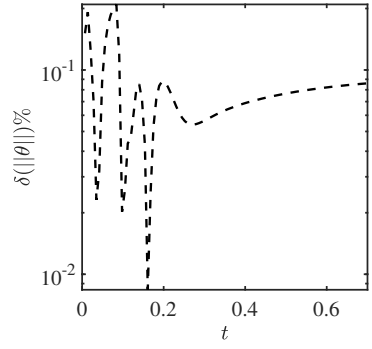

(e) $B=0.005$

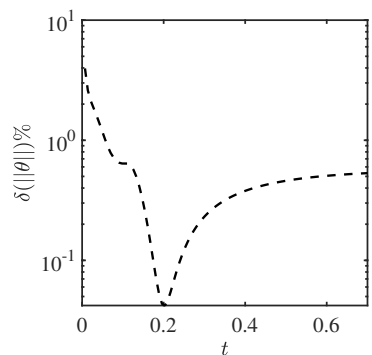

(g) $B=0.015$

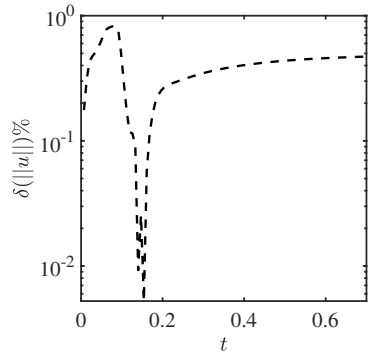

(b) $B=0.01$

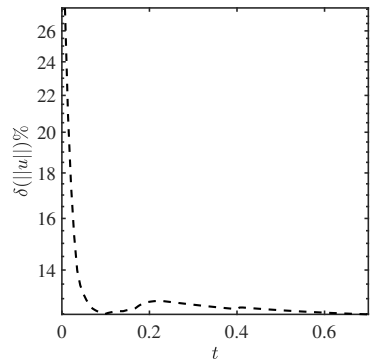

(d) $B=0.025$

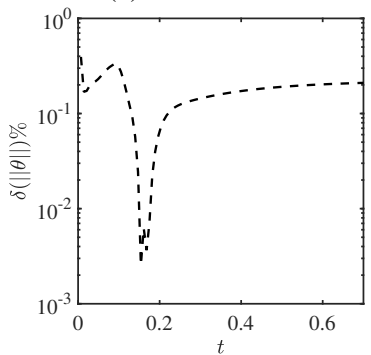

(f) $B=0.01$

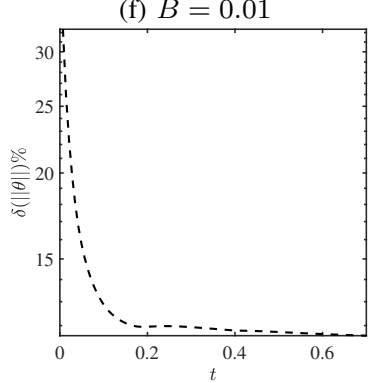

(h) $B=0.025$
Fig. 4. The evolution of (a - d) $\delta(\|u\|)$, and (e - h) $\delta(\|\theta\|)$ for different Bingham numbers at $R a=10^{4}$ and $\operatorname{Pr}=1$.

reveal what are the numerical difficulties associated with it. We tested a range of Bingham numbers in our numerical experiments to be able to determine when the regularized Bingham fluid starts to differ noticeably from the Bingham fluid. We utilized bi-viscosity model as a representative regularized model and assessed its accuracy against the augmented Lagrangian results.

We found that viscosity regularization predicts the dynamic and thermal unsteady behavior of the flow with a good accuracy for low to moderate Bingham numbers. For high Bingham numbers $\left(B \longrightarrow B_{c r}\right)$, nevertheless, the accuracy declines; we hypothesize that due to the small strain rate (i.e.

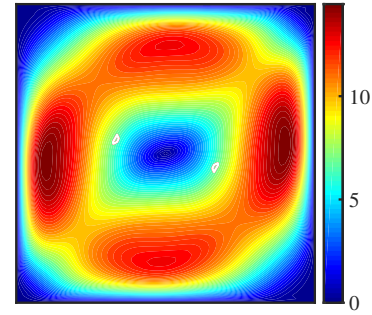

(a) $B=0.005$

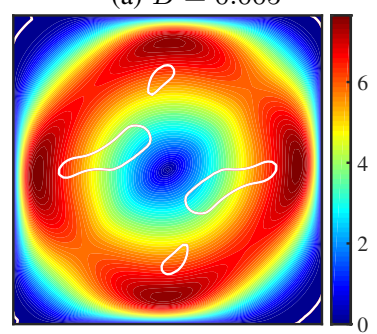

(c) $B=0.015$

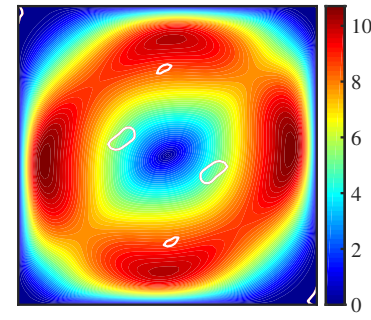

(b) $B=0.01$

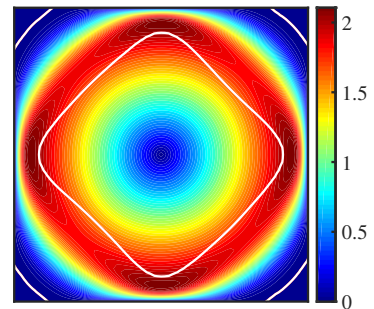

(d) $B=0.025$
Fig. 5. Colormaps of velocity for different Bingham numbers at $R a=10^{4}$ and $\operatorname{Pr}=1$; superimposed white closed curves show the domain cells with $\dot{\gamma} \leq \dot{\gamma}_{c r}$.

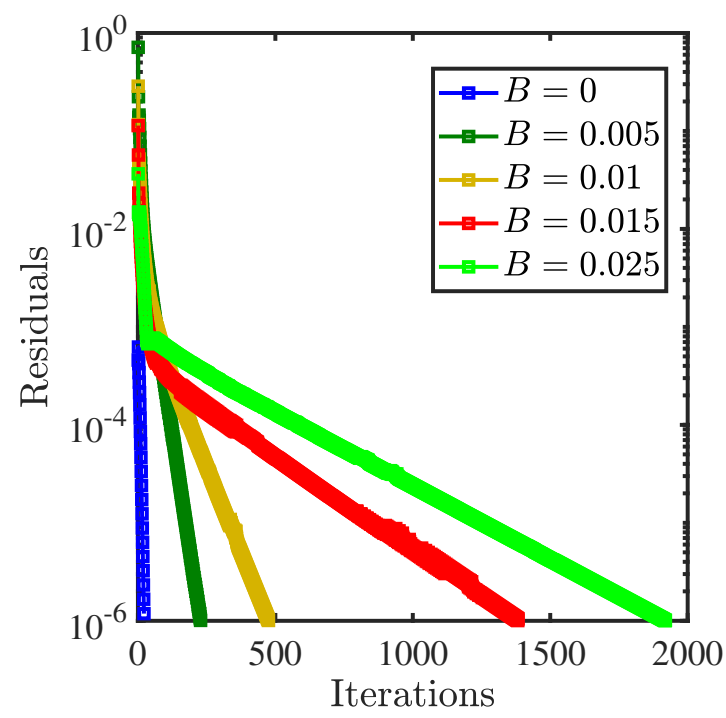

Fig. 6. Residual versus iteration at $t=0.0137$ for a biviscosity model with $m=1000$ with different Bingham numbers ramging from $B=0$ (Newtonian) to $B=0.025$ at $R a=10^{4}$ and $\operatorname{Pr}=1$

$\dot{\gamma} \leq \dot{\gamma}_{c r}$ ) in the domain, the regularization method fails to deliver an acceptable accuracy. Moreover, as we increase the Bingham number, the problem becomes numerically stiffer. Accordingly, the computational cost increases as more iterations are needed to achieve the desired precision. While the low computational cost of VR compared to $\mathrm{AL}$ is one of their main advantages, at high Bingham numbers $\left(B \longrightarrow B_{c r}\right)$, the computational cost increases significantly, undermining the commonly perceived value. 


\section{REFERENCES}

[1] N. J. Balmforth, I. A. Frigaard, and G. Ovarlez, "Yielding to stress: recent developments in viscoplastic fluid mechanics," Annual Review of Fluid Mechanics, vol. 46, pp. 121-146, 2014.

[2] D. Bonn, M. M. Denn, L. Berthier, T. Divoux, and S. Manneville, "Yield stress materials in soft condensed matter," Reviews of Modern Physics, vol. 89, no. 3, p. 035005, 2017.

[3] E. C. Bingham, Fluidity and plasticity, vol. 2. McGrawHill, 1922.

[4] R. Glowinski, Numerical methods for nonlinear variational problems. Springer-Verlag, New York, 1984.

[5] T. Treskatis, A. Roustaei, I. Frigaard, and A. Wachs, "Practical guidelines for fast, efficient and robust simulations of yield-stress flows without regularisation: A study of accelerated proximal gradient and augmented lagrangian methods," Journal of Non-Newtonian Fluid Mechanics, vol. 262, pp. 149-164, 2018.

[6] R. Glowinski, J.-L. Lions, et al., Numerical analysis of variational inequalities. Elsevier, 1981.

[7] M. Bercovier and M. Engelman, "A finite-element method for incompressible non-newtonian flows," Journal of Computational Physics, vol. 36, no. 3, pp. 313326, 1980.

[8] T. C. Papanastasiou, "Flows of materials with yield," Journal of Rheology, vol. 31, no. 5, pp. 385-404, 1987.

[9] R. Tanner and J. Milthorpe, "Numerical simulation of the flow of fluids with yield stress," Numer Meth Lami Turb Flow Seattle, pp. 680-690, 1983.

[10] I. Frigaard and C. Nouar, "On the usage of viscosity regularisation methods for visco-plastic fluid flow computation," Journal of non-newtonian fluid mechanics, vol. 127, no. 1, pp. 1-26, 2005.

[11] I. Karimfazli, I. Frigaard, and A. Wachs, "A novel heat transfer switch using the yield stress," Journal of Fluid Mechanics, vol. 783, pp. 526-566, 2015.

[12] O. Turan, N. Chakraborty, and R. J. Poole, "Laminar natural convection of bingham fluids in a square enclosure with differentially heated side walls," Journal of Non-Newtonian Fluid Mechanics, vol. 165, no. 15-16, pp. 901-913, 2010. 